\title{
Los docentes ante las demandas de las políticas de educación inclusiva para la atención de niños con diferentes discapacidades: casos de México y Brasil
}

Débora Dainez * Gabriela Naranjo * *

http://dx.doi.org/10.1590/0103-7307201507710

\section{Resumen}

Este artículo analiza la forma en que dos docentes de escuela regular, uno de México y otro de Brasil, respondieron ante las demandas de las políticas de educación inclusiva relacionadas con la atención de alumnos con discapacidad. El análisis de los casos permite evidenciar que los desafíos que dichas políticas imponen a los docentes no consideran las condiciones concretas en que desarrollan su labor, entre ellas, destaca la heterogeneidad de posibilidades en que puede presentarse la propia discapacidad, lo cual implica retos diferenciales para el desarrollo de la enseñanza. Aún así, los docentes asumen con un gran compromiso y desde su propia experiencia la responsabilidad de brindar una educación de calidad a estos alumnos, buscando activamente las estrategias más pertinentes para lograrlo.

Palabras-clave: educación inclusiva, trabajo de enseñanza, discapacidad, políticas educativas, educación básica
* Faculdade de Educação, Universidade Estadual de Campinas (Unicamp), Campinas, SP, Brasil. ddainez@yahoo.com.br

* * Secretaría de Educación Pública en México Asesora en la Dirección de Educación Especial, Distrito Federal, Mexico. gabriela.naranjo@prodigy. net.mx 


\title{
Teachers in the face of the educational inclusive policies: the cases of Mexico and Brazil
}

\begin{abstract}
This article analyzes how two teachers, one Mexican and another Brazilian, responded to the demands originated from inclusive policies regarding children with disabilities. The analysis shows that the challenges these policies impose on teachers do no take into consideration the concrete conditions under which they perform their work. In spite of those conditions, the teachers observed mobilized their own experience and their commitment for providing quality education to the students that were put under their responsibility. They did by this actively seeking the most relevant strategies to achieve it.
\end{abstract}

Keywords: inclusive education, teaching, disabilities, educational policies, basic education, Brazil and Mexico. 


\section{Introducción}

La atención educativa de los niños con deficiencia en Latinoamérica, si bien con sus especificidades, está enmarcada en un paquete de políticas inclusivas más amplias derivadas de una agenda global "propuesta” por organismos internacionales de distinto signo ideológico y difundidas a través de una diversidad de documentos y publicaciones (Buenfil, 2000; Krawczyk \& Vieira, 2012). Bajo un discurso hegemónico que parece atender legítimas demandas sociales, estas políticas son presentadas como vía para democratizar los sistemas educativos nacionales.

La agenda inclusiva para la educación tiene más de veinte años de haberse propuesto, no sin refinamientos y nuevos matices. A pesar de que los diferentes gobiernos de América Latina han impulsado políticas nacionales orientadas a la inclusión educativa de todos los niños independientemente de sus características y condiciones, poblaciones como la de quienes presentan discapacidad continúan sin recibir una educación que propicie su pleno desarrollo individual y social. Los discursos que por diferentes vías se están movilizando para justificar este fracaso están intensificando el argumento de la incompetencia de los docentes, se les acusa de mantener actitudes y concepciones excluyentes y de mantener una fuerte resistencia al cambio. En este trabajo, a partir del análisis de los casos de dos docentes, uno de México y otro de Brasil, pretendemos aportar elementos para poner en cuestionamiento estos argumentos.

Así, el propósito de este artículo es analizar la forma en que dos docentes de escuela regular, uno de México y otro de Brasil, respondieron ante las demandas de las políticas de educación inclusiva relacionadas con la atención de alumnos con discapacidad. Para ello, en la primera parte de este trabajo, destacamos las principales directrices de política internacional a partir de las cuales países como México y Brasil orientaron sus propias políticas de educación inclusiva, lo cual llevó, entre otras cosas, a que cada vez un mayor número de alumnos con discapacidad esté en las aulas comunes, con todos los retos y demandas que esto implica para los docentes. En la segunda parte presentamos los casos: un maestro atendiendo un grupo de escuela mexicana en el que está incluido un alumno ciego y una maestra atendiendo un grupo de escuela brasileña en el que se encuentra un alumno con discapacidad múltiple. En ambos casos, se trata de maestros que no recibieron capacitación específica ni contaron con apoyos adicionales significativos para trabajar con niños con discapacidad.

Presentar casos de dos países lationoamericanos permite resaltar lo común en la 
particularidad. Esto es, por un lado, que las políticas nacionales siguen en términos generales las mismas recomendaciones de política internacional para la atención de alumnos con discapacidad, y por otro, lo efectos comunes que ellas están teniendo en las demandas impuestas al trabajo de los docentes y las condiciones materiales en las que lo están haciendo.

\section{Una agenda global para la educación de niños con discapacidad}

A través del análisis de los documentos producidos en diferentes reuniones organizadas y financiadas por agencias multilaterales (tales como la UNESCO, OCDE, ONU, BM, CEPAL) y que fueron firmados por países como México y Brasil, es posible dar cuenta de las directrices generales que los gobiernos de estos países han asumido para orientar sus propias políticas inclusivas en el ámbito educativo. Si bien la influencia de agencias internacionales "se resignifican o cambian de sentido en las acciones mismas mediante las cuales se llevan a cabo en cada sitio particular" (Buenfil, 2000, p. 58), en los documentos y leyes emitidas por estos países es posible reconocer la marcada influencia de los discursos internacionales.

En este trabajo enfocamos el análisis en tres documentos que consideramos especialmente significativos por su relación con la atención educativa de niños con discapacidad. Se trata de la Declaración Mundial de Educación para Todos, producto de la Conferencia Mundial de Educación para Todos realizada en Jomtién, Tailandia en 1990; la Declaración de Salamanca, producto de la Conferencia Mundial sobre Necesidades Educativas Especiales, realizada en Salamanca, España, en 1994; y la Convención sobre los Derechos de las Personas con Discapacidad, realizada en Nueva York en 2006.

\section{La Declaración Mundial de Educación para Todos}

De acuerdo con Pletsch (2010), la llamada educación inclusiva tiene su inicio en la década de 1990 con la política de universalización de la educación básica prevista en la Declaración Mundial de Educación para Todos.

Al recomendar promover una educación para "todos" se incluye a un espectro muy amplio y abstracto de personas, sin embargo, es en ciertos grupos que se pone el énfasis: las niñas y mujeres, los pobres, niños de la calle y los niños que trabajan; las poblaciones de zonas remotas y rurales; los nómadas y trabajadores migrantes; los pueblos indígenas; las minorías étnicas, raciales y lingüísticas; los refugiados, los desplazados por la guerra y los pueblos sometidos a un régimen de ocupación. Como 
un grupo de especial atención entre los desfavorecidos se destaca el de las personas con discapacidad.

Bajo el principio de “Educación para Todos” y con el objetivo de satisfacer las necesidades básicas de sus ciudadanos, los gobiernos de los países firmantes de la Declaración se dieron a la tarea de reformar sus sistemas educativos, de hecho México ya estaba realizando las primeras acciones con su Programa de Modernización Educativa (SEP, 1989). Con la firma del Acuerdo Nacional para la Modernización de la Educación Básica $(S E P, 1992)$ y la promulgación de la nueva Ley General de Educación (SEP, 1993) se dieron pasos firmes hacia el cumplimiento, por lo menos en la ley, de los compromisos asumidos en Jomtién. En el caso de Brasil, las acciones orientadas a reformar su sistema educativo iniciaron a finales de la década de los 80 con la homologación de la Constitución Federal (Brasil, 1988), por la cual la educación es presentada como un derecho social. En lo que se refiere a las personas con discapacidad se le da prioridad a su atención en la red pública de educación. Según Kassar (2011), a partir de 1988, la política educativa en Brasil pasó a ser de carácter universal con diferentes efectos en el sistema de enseñanza.

Para Garcia (2008), la idea de universalización de la educación básica ganó fuerza no sólo porque expresaba una demanda la sociedad sino también porque era una condición necesaria para mantener los contratos entre agencia financieras internacionales y los Estados nacionales. En el mismo sentido, Pletsch (2010), plantea que la Declaración de Jomtién está asociado a un proyecto neoliberal previsto por los organismos internacionales, tales como el Banco Mundial, que reconoce los derechos individuales, pero que no se responsabiliza frente a las condiciones que producen las desigualdades sociales. En lugar de ello, se coloca a la educación básica como la estrategia más viable para reducir la pobreza, prevenir los problemas sociales, lograr la movilidad social, aspirar a un buen empleo y calidad de vida, mejorar la salud, etc.

Así, el ingreso y la permanencia de los individuos en los sistemas educativos se presenta como el medio para disminuir las desigualdades, enmascarando las necesidades concretas de inversión pública para hacer efectivas las políticas sociales y de educación.

\section{La Declaración de Salamanca}

El discurso de la Conferencia de Educación para Todos fue discutido, traducido y desdoblado en el ámbito de la educación especial, principalmente a través la realización de la Conferencia Mundial sobre Necesidades Educativas Especiales, realizada 
en Salamanca España, en 1994 y auspiciada por la UNESCO, el UNICEF, el PNUD y el Banco Mundial. La importancia de este evento radica en que concretó el consenso internacional para la orientación de la educación especial y para la atención educativa de los alumnos con discapacidad, además, a través de ella la UNESCO inició de manera más formal la llamada por la "inclusión educativa” (Garcia, 2008).

En la Declaración de Salamanca se delega a las escuelas y sus maestros la responsabilidad, no sólo de “dar una educación de calidad a todos los niños”, sino de "intentar cambiar las actitudes de discriminación, crear comunidades que acojan a todos y sociedades integradoras." (Unesco, 1994, p. 6). De este modo, el discurso de la educación inclusiva atribuye amplios poderes a la escuela, los cuales van, desde el combate a las actitudes discriminatorias hasta la construcción de una sociedad integradora, delegándole además la responsabilidad por la transformación de la realidad social. El supuesto de base es que aún en las condiciones adversas del contexto económico-político-ideológico, la escuela tiene una función que, ejercida de forma competente, debe posibilitar la apropiación del saber por todos los ciudadanos (Jannuzzi, 2006; Laplane, 2004).

Basada en concepto de necesidades educativas especiales, en la Declaración de Salamanca se cuestiona el énfasis puesto en el "déficit" del niño y se señala la incapacidad de los sistemas de educación para satisfacer los requerimientos especiales de educación de los alumnos ya sea que presenten o no discapacidad; se cuestiona también la práctica de mantener dos sistemas de enseñanza: el regular y el especial. Así, el discurso de esta declaración orientó a los países firmantes hacia la unificación de la educación especial con la educación “ordinaria” o "regular”. El argumento del alto costo y baja eficiencia de las escuelas especiales fue uno de los que mejor justificaron en diversos países, incluidos México y Brasil, la adopción de políticas inclusivas como estrategia para lograr la sustentabilidad financiera.

La Declaración de Salamanca recomienda impulsar escuelas integradoras, ya que ellas "representan un marco favorable para lograr la igualdad de oportunidades y la completa participación” (Unesco, 1994, p. 11) y para la inclusión de los niños con necesidades educativas especiales. Bajo este discurso se advierte una crítica al carácter “excluyente” de las escuelas, como si la exclusión no fuera más bien una característica esencial de los sistemas y dinámicas socioeconómicos de los países capitalistas. Así, se justifica la necesidad de impulsar la transformación de las escuelas, imponiéndoles con esto una serie de demandas y en especial a sus maestros, tales como ser 
capaces de "desarrollar una pedagogía centrada en el niño, capaz de educar con éxito a todos los niños y niñas, comprendidos los que sufren discapacidades graves". Esto implica que "deben reconocer las diferentes necesidades de sus alumnos y responder a ellas, adaptarse a los diferentes estilos y ritmos de aprendizaje" y que tienen que dar a los niños con necesidades educativas especiales "todo el apoyo adicional necesario para garantizar una educación eficaz” (Unesco, 1994, pp.11-12). La crítica que parece estar detrás de estas orientaciones es que los maestros organizan su enseñanza sin considerar quiénes son sus alumnos y cuáles son sus necesidades y que son incapaces o muestran poca disposición para solventar las dificultades de los niños en sus propias aulas, ya que muchas de ellas se podrían evitar si implementaran formas más adecuadas de enseñanza.

De esta manera a los profesores se les asigna la responsabilidad de atender la diversidad y alcanzar "los niveles adecuados de aprendizaje" con todos los alumnos, mas no se aborda la cuestión de cuáles son las condiciones concretas en las que debiera desarrollarse el trabajo pedagógico.

Del análisis realizado destaca que una serie de cuestionamientos a los sistemas educativos en general, a la educación especial y a las escuelas y sus maestros en partícular fueron abonando argumentos para definir la agenda de educación inclusiva. Así, la Declaración de Salamanca concreta una serie de directrices y orientaciones para la reforma de los sistemas educativos y el “reacomodo" de la educación especial en ellos.

En el caso de México, la educación especial deja de funcionar como un sistema paralelo, para constituirse en una modalidad de la educación básica y adopta a la integración "como un programa de desarrollo institucional y como un espacio para ofrecer calidad educativa a todos los educandos" (DEE/SEP, 1994). Así los servicios de educación especial quedaron distribuidos en dos modalidades, una constituida por servicios de apoyo a la escuela regular, cuya principal función sería favorecer la integración educativa de los alumnos con necesidades educativas especiales con o sin discapacidad y a aquellos con capacidades sobresalientes en las escuelas comunes. Y la otra constituida por escuelas cuya función sería la de preparar a los niños para ser integrados a la escuelas regulares.

Al igual que en México, en Brasil la educación especial pasó a ser tratada como una modalidad

1. Nótese que en México el término "integración” se adoptó como la palabra clave para definir la política de la educación especial en los 90's, mientras que en Brasil, fue más bien el de "inclusión”. Cuando asumieron y firmaron la Declaración de Salamanca ambos países ya habían desarrollado experiencias de integración educativa, a partir de las cuales cada uno de ellos desarrolló sus propias interpretaciones y definió sus formas específicas de concretar las recomendaciones de dicho documento. 
de la educación común y, con ello, se establecieron dos servicios: uno de apoyo a la escuela regular y otro de escolarización, teniendo como base un currículo y formas de evaluación comunes. Destaca en el año de 1996 la aprobación de la nueva Ley de Direcrices y Bases para la Educación (Brasil, 1996), que incluye un capítulo dedicado a la educación especial en el que se define la oferta de atención a los alumnos en escuelas comunes basado en las necesidades educativas especiales. Esto desencadenó, según Ferreira (2006), en la aceleración del proceso de municipalización de la educación básica y en la concentración del 60\% de los recursos presupuestales de la educación en ese nivel educativo, llevando a una expansión acentuada de acceso de los alumnos con discapacidad a las series iniciales.

De esta forma, tanto en Brasil como en México, la década de los noventa estuvo marcada por la implantación de acciones gubernamentales en varias áreas de los servicios públicos, entre ellos la educación, como parte de las llamadas políticas de inclusión social que trae inherente la idea de una población excluida. La propuesta de una educación inclusiva en la década siguiente llegó a tener una vasta circulación, aunque aún sin concretar las condiciones para hacerla efectiva.

\section{La Convención sobre los Derechos de las Personas con Discapacidad}

La Convención sobre los Derechos de las Personas con Discapacidad realizada en 2006 en Nueva York es el documento que de forma más reciente concreta las recomendaciones para impulsar políticas de inclusión de las personas con discapacidad en diferentes ámbitos de la vida social.

Si bien el propósito declarado en la Convención es garantizar que las personas con discapacidad accedan a la educación y con ello a la posibilidad de aprender a lo largo de la vida, tal garantía queda debilitada cuando en ella se prevé la realización de “ajustes razonables en función de las necesidades individuales”. Con esta previsión, el discurso deja un intersticio abierto por el cual se torna posible que los gobiernos de los países firmantes con sus sistemas educativos puedan evadir su responsabilidad de realizar los cambios necesarios para que el derecho a la educación, tan vehementemente reconocido, se haga efectivo. El concepto de ajustes razonables, plagado de una gran ambigüedad, es el que deja abierto ese intersticio:

Por "ajustes razonables" se entenderán las modificaciones y adaptaciones necesarias y adecuadas que no impongan una carga desproporcionada o indebida ...para garantizar 
a las personas con discapacidad el goce o ejercicio, en igualdad de condiciones con las demás, de todos los derechos humanos y libertades fundamentales (0NU, 2006, p. 5).

Los ajustes razonables hacen referencia a modificaciones o adaptaciones "que no impongan una carga desproporcionada o indebida". Pero ¿quién, cómo y en qué circunstancias se define lo que es razonable o no, lo que representa una carga desproporcionada o indebida?, ¿desproporcionada o indebida para qué o para quién? Así, la propuesta de educación inclusiva no habla de transformaciones significativas en los sistemas educativos, en las estructuras de las escuelas, sólo habla de modificaciones que les sean "posibles". Con ello, se "suaviza" la responsabilidad de los Estados nacionales en la generación de las condiciones necesarias para garantizar el cumplimiento del derecho a la educación de las personas con discapacidad.

Al ser México uno de los promotores de la Convención, el compromiso firmado se tradujo en la publicación de una Ley General para la Inclusión de las Personas con Discapacidad en el 2011. En ella el Estado se compromete a promover, proteger y asegurar el pleno ejercicio de los derechos humanos y libertades fundamentales de estas personas, con un capítulo dedicado específicamente a la educación, donde se dispone hacer lo necesario para garantizar el goce de este derecho y para impulsar su inclusión en todos los niveles del Sistema Educativo.

Los efectos de la Convención en Brasil se observan en los dos últimos decretos emitidos. El primero de ellos, el 7.611 (Brasil, 2011a), hace referencia a la educación especial que, entre otras cosas, contempla la atención educativa especializada; y el segundo, el 7.612 (Brasil, 2011b), instituye el Plan Nacional de los Derechos de las Personas con Deficiencia-Plan Vivir sin Límite. Esos decretos contemplan atender todas las dimensiones de la vida de la persona, garantizar el aprendizaje continuo a lo largo de la vida y hacen responsable al sistema educativo en general por la persona con deficiencia. Sin embargo, un aspecto importante a destacar es el manejo que se hace en la ley de las barreras educativas. Se reconoce que éstas no se relacionan con la discapacidad del alumno, lo cual es un avance significativo, pero se infiere que aluden al "proceso de enseñanza y aprendizaje”, enfocándose en la relación profesor-alumno. Es decir, no se contempla que los obstáculos y barreras para la inclusión educativa se forjan también en las condiciones en que se produce el proceso de enseñanza y aprendizaje. 
No está dentro de los propósitos de este texto analizar las formas específicas en que las directrices internacionales se fueron concretando en México y Brasil. Sólo se ha hecho mención a las medidas políticas y legales más generales que fueron tomadas por cada gobierno para cumplir con los compromisos asumidos al firmar los documentos referidos. Lo que nos interesa en la siguiente parte de este trabajo es analizar de manera más directa lo que ocurre en las aulas, que es finalmente donde se reflejan los efectos de las políticas inclusivas. En relación con esto, en ambos países destaca la presencia cada vez más frecuente de alumnos con discapacidad en los grupos atendidos por maestros comunes en escuelas comunes. En este sentido, se observa que una de las pretensiones de las políticas inclusivas se va cumpliendo, el que los niños con discapacidad estén en las aulas regulares. Pero sabemos bien que eso no basta, la cuestión es ver qué sucede ahí, cómo se concreta la educación de estos pequeños, cómo los docentes van afrontando las demandas que las políticas inclusivas les imponen en su trabajo cotidiano.

La relación que se hace entre Brasil y México a partir de dos casos no tiene la intención de evaluar cuál ha avanzado más o menos o cuál ha tenido mejores resultados, se trata más bien de destacar, a partir de algunas categorías de análisis los retos a los que se tienen que enfrentar los maestros en su trabajo cotidiano como efecto de las políticas inclusivas, las formas en que lo están haciendo y algunas de las condiciones objetivas que tienen para ello.

La confrontación de los casos, a través de un intercambio académico intenso entre las investigadoras, permitió construir el objeto de estudio relacionado con las condiciones en que los docentes implementan las políticas de educación inclusiva en sus aulas. En ambos casos la etnografía fue la perspectiva metodológica que orientó las indagaciones y el análisis de los datos. Desde esta perspectiva, buscamos descifrar el significado de la acción social, analizarla en su especificidad y circunstancialidad e indagar lo que dicha acción nos dice respecto de procesos más amplios (Geertz, 2008). Se hicieron observaciones de clases y de los contextos áulicos y escolares en cada caso, así como entrevistas formales e informales a los maestros de grupo.

\section{Los maestros ante las demandas de las políticas inclusivas}

Conforme las políticas inclusivas se han ido desarrollando y sofisticando, la cantidad y tipo de demandas a las escuelas y, sobre todo, a sus maestros se intensifican, con sus consecuentes efectos en el proceso de enseñanza aprendizaje y en las posi- 
bilidades de desarrollo de los niños con discapacidad. En este apartado destacamos cómo los maestros en los casos analizados han hecho frente a dichas demandas.

\section{¿Alumnos con discapacidad?... ¿qué discapacidad?}

Una primera demanda para los docentes por las políticas inclusivas es que ellos deben satisfacer las necesidades básicas de aprendizaje y las necesidades educativas especiales de todos y cada uno de los niños y niñas, lo cual implica que deben conocer y saber lidiar con cualquier situación de desarrollo en que un niño pudiera llegar a ser parte de su grupo. Como se verá enseguida, los casos que analizamos implican a dos niños que presentan condiciones de discapacidad bastante contrastantes.

\section{Toño, un alumno con déficit sensorial}

Toño, de 10 años de edad, es un alumno de cuarto grado en una escuela primaria mexicana que presenta ceguera congénita. Cursó su educación preescolar en una escuela pública ordinaria y ha estado en la misma escuela primaria desde primer grado sin haber reprobado. Toño mantiene una buena relación con sus compañeros y maestro, goza del aprecio y reconocimiento de la comunidad educativa. Generalmente parece estar alegre y alerta a lo que sucede a su alrededor, se desplaza sin dificultad por el salón de clases a excepción de las ocasiones en que hay algo nuevo en él (por ejemplo, la cámara de vídeo), en cuyo caso el maestro le previene y le indica por donde caminar.

\section{André, un alumno con Síndrome de Angelman}

André es un alumno con 11 años de edad de quinto grado en una escuela primaria de Brasil que presenta deficiencia múltiple como secuela de Síndrome de Angelam, un disturbio genético-neurológico caracterizado por retraso en el desarrollo intelectual, alteraciones en el habla, disturbios del sueño, convulsiones, movimientos desconexos y risas frecuentes y descontextualizadas. André no habla y realiza pocos movimientos voluntarios de miembros superiores e inferiores. Usa silla de ruedas acolchonada a los lados, con chaleco pectoral y una mesa acoplada. Toma dosis altas de medicamentos para tratar las crisis convulsivas. Asiste a la escuela desde el primer grado. A André se le fue promoviendo de grado escolar bajo el criterio de edad, sin que hubiera alcanzado los conocimientos y habilidades necesarias para acompañar las actividades pedagógicas. 
Tanto Toño como André pueden ser considerados dentro del grupo de "personas con discapacidad", sin embargo, la especificidad de la deficiencia que cada uno presenta implica condiciones de desarrollo muy distintas entre sí y retos diferenciales para las escuelas y sobre todo para los maestros. Mientras que para brindar educación y potenciar el desarrollo de un alumno con ceguera existen recursos históricamente producidos (por ejemplo, un sistema de comunicación escrito como el Braille) y un cierto conocimiento socialmente compartido, para un alumno con una deficiencia múltiple y severa, ocasionada por un síndrome aún poco conocido y estudiado, no se tienen prácticamente herramientas para su atención pedagógica. No obstante lo anterior, cada maestro debe ser capaz de trabajar con cualquier condición (sordera, ceguera, sordo-ceguera, deficiencia intelectual, autismo, discapacidad múltiple, entre otras), esto es así porque desde las políticas de educación inclusiva, las diferentes condiciones discapacidad son tratadas como si fueran equivalentes.

\section{Diferentes maestros, mismas actitudes ante sus alumnos con discapacidad}

Como se analizó en el discurso de los documentos, bajo el supuesto de que muchos maestros ejercen prácticas segregacionistas, se les exige un cambio de actitudes y concepciones hacia los educandos con requerimientos especiales. De acuerdo con esto, se advierte que una de las mayores dificultades para avanzar hacia una educación inclusiva son las resistencias por abolir toda forma de discriminación, las mismas que han generado prácticas excluyentes en grupos escolares y maestros y que una de las tareas más fuertes es trabajar sobre estas barreras ideológicas y conceptuales (Arroyo, 1997).

A lo largo de este análisis se irán evidenciando ciertas actitudes y concepciones de los maestros en los casos que se presentan. Por lo pronto, a modo de ejemplo, citamos lo que el maestro de Toño, dijo respecto a su alumno:

Al principio, cuando me dijeron que iba a tener en mi grupo a este niño, sentí temor, no al niño sino a lo desconocido, pero me bastaron dos días para darme cuenta que mi Toñito es un niño como los demás, de hecho trabajo con él igual que con los demás, sin darle ventajas ni desventajas, porque no se trata de hacerlo dependiente, sino de enfrentarlo a la vida (Diario de campo, caso México).

La visión que el maestro expresa sobre su alumno se refleja en su opinión después 
de varios meses de trabajo con él: “El niño es muy bueno, es listo, es de los mejores de la clase, incluso mejor que los demás, en el primer bimestre sacó el primer lugar, en el segundo me bajó un poco, pero va bien" (Diario de campo, caso México). El maestro reporta que Toño lee mucho, que eso es algo que le favorece y reconoce su gran potencial por lo que trata frecuentemente de implicarlo en actividades diversas, como por ejemplo, en las ceremonias cívicas y en los paseos escolares.

Por su parte, la condición de desarrollo de André, impone a su maestra desafíos de una gran complejidad, provocándole, no sin razón, incertezas y desconciertos: “André está siendo un descubrimiento. Yo no tengo certeza si él comprende o no, mas creo que él me puede comprender. Por ejemplo, cuando digo su nombre él me mira y sonríe" (Diario de campo, caso Brasil).

Aún con sus dudas, la profesora cree que André comprende, lo cual, por un lado, exhibe las inseguridades que el reto de brindarle una educación le generan, pero, por otro lado, muestra una expectativa y actitud favorable hacia su alumno, lo que la lleva a buscar formas diferentes de interacción y de intervención educativa con él. André, siendo una incógnita para la maestra, es colocado por ella en el lugar de quien puede participar de un mundo simbólico.

Contrario a los cuestionamientos implícitos en los discursos de las políticas, tanto la profesora de André, como el profesor de Toño creen en las posibilidades de sus alumnos, muestran actitudes favorables hacia ellos y desarrollan sus acciones educativas en consecuencia.

\section{Los esfuerzos docentes por una educación inclusiva}

En el plano pedagógico, las políticas de educación inclusiva demandan a los maestros buscar, crear, encontrar, generar métodos, estrategias, formas de trabajo que permitan potenciar el desarrollo y aprendizaje de los niños sea cual sea su condición, incluidos aquellos con discapacidades graves. En los casos de análisis veamos qué es lo que los maestros hacen efectivamente tratando de enfrentar este reto.

\section{Enseñar ciencias naturales a un grupo con un alumno ciego}

La observación y análisis de clases de ciencias naturales, enfocada en momentos de realización de actividades experimentales, permitió aproximarse a la forma en la que este maestro trataba de hacer efectivo el derecho de su alumno ciego a la educación. Destaca en primer lugar la implementación de algunas estrategias sencillas que, 
sin embargo, expresaban una preocupación constante por lograr que su alumno participara de la dinámica del aula. Por ejemplo, para trabajar la lección se sentaba junto a él y de cara a todo el grupo, ya que de esta manera, según el propio maestro, podía brindarle apoyo cuando lo necesitara y verificar que ponía atención a lo que se estaba trabajando. Esto se constató al observar que mantenía frecuentes intercambios verbales con él para retroalimentarlo, llamarle la atención, escuchar sus experiencias extraescolares y responder a sus preguntas. Por otro lado, el maestro promovía que sus compañeros lo apoyaran en tareas que lo requerían, que trabajaran con él en equipo, le compartieran su material y le describieran lo que observaban durante las actividades experimentales.

En segundo lugar, el maestro implementaba formas de trabajo que consideraban las posibilidades de su alumno ciego para acceder a los contenidos abordados. De esta manera, frecuentemente recurría a la generación de experiencias físicas que le proporcionaran referentes no visuales para tener una mejor comprensión de los fenómenos naturales bajo estudio. Por ejemplo, en una sesión de trabajo colectivo, después de leer la siguiente pregunta "¿Qué crees que sucede cuando se agrega un cubo de hielo a un vaso con refresco?" y de que el grupo responde en coro "se derrite", el maestro interrumpe la lectura para demostrar esto a Toño empíricamente. Dice a todo el grupo: "eso ya lo saben ustedes, pero lo vamos a hacer para que Toño lo toque". Por otro lado, el trabajo con los contenidos y, específicamente, la realización de las actividades experimentales era constantemente acompañado con descripciones verbales detalladas por parte del maestro. Así, el habla apareció como uno de los recursos más poderosos mediante los cuales el docente trataba de aprovechar la experiencia física vivida por su alumno ciego y compartir con él aquella que visualmente no le era accesible.

\section{La apuesta por el aprendizaje de un alumno en condiciones de discapacidad severa}

Al principio la maestra desarrollaba su trabajo con André siguiendo las orientaciones contenidas en un texto dejado a la escuela por especialistas en el área de la salud: darle masajes en los miembros superiores e inferiores, estimulación sonora, visual, táctil con diferentes objetos (juguetes, un paño, un vaso). Así, entre las acciones que ella realizaba estaban por ejemplo, mover un paño por atrás de la cabeza de André para que éste lo siguiera con la mirada; ponerlo cerca de la puerta para que mirara a otros niños moviéndose durante el recreo afuera del salón de clases; golpear la puerta 
del aula varias veces para atraer su mirada. También lo dejaba dormir un buen tiempo de la jornada, ya que los medicamentos que André tomaba le provocaban sueño.

A medida que la profesora se fue aproximando y conociendo más al alumno, fue cambiando su modo de trabajar con él. En primer lugar, comenzó a hacer una trabajo de interpretación de los movimientos y expresiones del alumno, atribuyendo significado y nombrando sus acciones y sus gestos (sonrisas, quejidos, sueño, señas de adiós). Además empezó a conversar con él sobre situaciones vividas en el aula y a procurar mantenerlo despierto en el aula por más tiempo.

En segundo lugar, si bien los contenidos específicos de las asignaturas no eran trabajados de manera específica con André, la profesora intentaba aproximarlo a situaciones que pudieran propiciarle algún aprendizaje. Así, sentaba al alumno junto a un grupo de colegas que realizaban las actividades académicas propuestas, promovía el apoyo de sus compañeros al pedirles que le platicaran o mostraran aspectos sobre las actividades que estaban desarrollando. En otros momentos, la profesora paseaba con André en su silla de ruedas por el salón de clases, parando en las mesas de los demás alumnos y mostrándole la actividad de sus colegas. Por ejemplo, en una situación en la que el grupo veía un vídeo sobre la Mata Atlántica, la profesora se sentó junto a André para tratar de orientar la atención de André hacia el video de varias formas: ayudándole a sostener el tronco, señalando hacia la TV y haciendo comentarios sobre el contenido del video.

Lo que se pudo observar en ambos casos es el interés de los docentes por hacer que sus alumnos con discapacidad participaran de la vida social y académica del aula. Ellos definieron sus acciones pedagógicas considerando las condiciones de sus alumnos, tratando de potenciar sus posibilidades, aún cuando, como en el caso de André, la docente no tuviera muchas certezas y escasos recursos. De ahí que podemos decir que en estos casos, los maestros de ambos alumnos, con condiciones tan diferentes, enfrentaron las demandas de la política con todo el compromiso y la responsabilidad que les fue posible. Los breves relatos del trabajo de estos maestros con sus alumnos con discapacidad muestran los esfuerzos que realizaron por buscar, ensayar y crear posibles formas de educarlos.

\section{Consideraciones finales}

El propósito de este trabajo fue analizar la forma en que dos docentes de escuela regular, uno de México y otro de Brasil, respondieron ante las demandas de las políticas de educación inclusiva relacionadas con la atención de alumnos con discapacidad. De esa discusión destacan algunas tensiones. Por un lado, las políticas de inclu- 
sión que implícitamente depositan en las escuelas y en sus profesores una gran parte de la responsabilidad por el cambio y la justicia social, sin considerar de manera significativa las condiciones objetivas en que la labor de enseñanza tiene que desenvolverse. Por otro lado, en el discurso de esos documentos, los profesores son colocados en el lugar del no-conocimiento, de la poca voluntad, de quien no tiene el suficiente compromiso pedagógico, del que tiende a excluir y del que precisa desarrollar actitudes, competencias y habilidades para poder incluir alumnos excluidos.

En los casos presentados de dos países latinoamericanos distintos encontramos maestros que no se corresponden con aquellos a los cuales los discursos políticos de los documentos analizados parecen aludir. Por el contrario, encontramos que estos dos profesores exhiben un gran compromiso ético y pedagógico con sus alumnos con discapacidad, que creen en sus potencialidades y que hacen muchos esfuerzos por crear situaciones que estimulen su desarrollo. Esto en un contexto de condiciones adversas para la realización de su trabajo docente (falta de capacitación, falta de recursos materiales y didáticos específicos, falta de apoyos que sustenten ese quehacer pedagógico, etc.).

Consideramos que los casos de alumnos con diferentes condiciones orgánicas (ceguera y deficiencia múltiple), presentados en perspectiva y en contrapunto en este estudio desafían el modelo educativo único y gerencial ofrecido por las políticas de educación inclusiva, que tienden a reunir en un solo "paquete” condiciones heterogéneas de desarrollo y humanización. Las especificidades del modo de constitución del individuo son diluidas en el grupo de los desfavorecidos y discapacitados. El contraste entre el caso de Toño (deficiencia sensorial) y el caso de André (deficiencia múltiple) nos posibilita ver lo que fue construido a lo largo de la historia y está disponible en términos de recursos. Toño y su profesor, aún en las condiciones precarias de educación inclusiva, están más amparados por lo ya producido en términos de conocimiento humano sobre la deficiencia visual y los recursos que eso generó, por ejemplo, el sistema Braille. Por su parte, André y su profesora, además de las condiciones difíciles de trabajo ya citadas, se enfrentan con la falta de recursos pedagógicos, didácticos y materiales, debido al desconocimiento que se tiene sobre el Síndrome de Angelman.

De este modo llamamos la atención hacia la naturaleza de la deficiencia como condición del trabajo docente, contraponiéndonos así a los documentos que igualan las discapacidad, que demandan sólo ajustes razonables, previendo inversiones precarias (económicas, entre ellas) en la educación de estas personas. 


\section{Referências bibliográficas}

Arroyo, M. A. (1997). Pensar la calidad de la atención especial desde la dimensión humana de los sujetos. Revisa Básica, 4 (16), 6-12.

Brasil (1988). Constituição da República Federativa do Brasil. Brasília: Brasil.

Brasil (1996). Lei de Diretrizes e Bases da Educação Nacional, n. 9.394. Brasília: Brasil.

Brasil (2011a). Decreto n. 7.611. Dispõe sobre a educação especial, o atendimento educacional especializado e dá outras providências. Diário Oficial da União, Brasília: Brasil.

Brasil (2011b). Decreto n. 7.612. Institui o Plano Nacional dos Direitos da Pessoa com Deficiência - Plano Viver sem Limite. Diário Oficial da União, Brasília: Brasil.

Buenfil, R. N. (2000). Globalización y políticas educativas en México 1988-1994. Encuentro de lo universal y lo particular. Revista Latinoamericana de Estudios Educativos, 6, 55-92.

Dirección de Educación Especial (DEP), SEP. (1994). Proyecto General para la Educación Especial en México. Cuadernos de Integración Educativa. México: DEE/SEP.

Ferreira, J. R. (2006). Educação especial, inclusão e política educacional: notas brasileiras. In D. Rodrigues (Org.), Inclusão e educação: doze olhares sobre a educação inclusiva (pp.85-113). São Paulo: Summus.

Garcia, R. M. C. (2008). Políticas inclusivas na educação: do global ao local. In C. Bap tista, K. Caiado, \& D. Jesus (Orgs.), Educação especial: diálogo e pluralidade (pp.11-23). Porto Alegre: Mediação.

Geertz, C. (2008). Descripción densa: hacia una teoría interpretativa de la cultura. In C. Geertz, La interpretación de las culturas (pp.19-40). Barcelona: Gedisa.

Jannuzzi, G. S. de M. (2006). A educação do deficiente no Brasil: dos primórdios ao início do século XXI (2a ed., p. 242). Campinas, SP: Autores Associados.

Kassar, M. de C. M. (2011). Percursos da constituição de uma política brasileira de educação especial inclusiva. Revista Brasileira de Educação Especial (edição especial), 17, 41-58.

Krawczyk, N. R., \& Vieira, V. L. (2012). Uma perspectiva histórico-sociológica da Re forma Educacional na América Latina. Argentina, Brasil, Chile e México nos anos 1990 (2a ed., p. 162). Brasilia: Liberlivros.

Laplane, A. L. F. de. (2004). Notas para uma análise dos discursos sobre inclusão escolar. In M. C. R. de Góes, \& A. L. F de Laplane (Orgs.), Políticas e Práticas de Educação Inclusiva (pp. 05-20). Campinas, SP: Autores Associados. 
Organização das Nacões Unidas (2006). Convention on the Rights of Persons with Disabilities. New York: ONU.

Pletsch, M. D. (2010). Repensando a inclusão escolar: diretrizes políticas, práticas curriculares e deficiência intelectual (p.280). Rio de Janeiro: Nau: Edur.

Secretaría de Educación Pública (1989). Programa de Modernización Educativa. México: SEP.

Secretaría de Educación Pública (1992). Acuerdo Nacional para la Modernización de la Educación Básica. México: SEP.

Secretaría de Educación Pública. (1993). Ley General de Educación. México: SEP.

Unesco (1994). Declaración de Salamanca y Marco de Acción para las Necesidades Educativas Especiales. Salamanca, España: Unesco.

Submetido à avaliação em 14 de fevereiro de 2014; aceito para publicação em 5 de setembro de 2014 . 